\title{
Suicidality and Depression Disparities between Sexual Minority and Heterosexual Youth: A Meta-Analytic Review
}

\author{
Michael P. Marshal, Ph.D.a,b,c, Laura J. Dietz, Ph.D. ${ }^{b}$, Mark S. Friedman, Ph.D. ${ }^{a, d}$, Ron Stall, \\ Ph.D. ${ }^{a, d}$, Helen Smith, Ph.D. ${ }^{a, e}$, James McGinley, B.A. ${ }^{\dagger}$, Brian C. Thoma, B.A.g, Pamela J. \\ Murray, M.D., M.H.P. ${ }^{h}$, Anthony D'Augelli, Ph.D. ${ }^{i}$, and David A. Brent, M.D. ${ }^{b}$ \\ aCenter for Research on Health and Sexual Orientation, Graduate School of Public Health, \\ University of Pittsburgh \\ b Department of Psychiatry, School of Medicine, University of Pittsburgh \\ c Department of Pediatrics, School of Medicine, University of Pittsburgh \\ d Department of Behavioral and Community Health Sciences, Graduate School of Public Health, \\ University of Pittsburgh \\ e Division of General Internal Medicine, School of Medicine, University of Pittsburgh \\ ${ }^{f}$ Department of Psychology, University of North Carolina, Chapel Hill \\ g Department of Psychology, University of Utah \\ h School of Medicine, West Virginia University \\ i Department of Human Development and Family Studies, The College of Health and Human \\ Development, Pennsylvania State University.
}

\section{Introduction}

Suicide is the third leading cause of death among adolescents and young adults in the United States, with lifetime prevalence rates ranging from 1-10\% in adolescents [1-3]. Following a decade of steady decline, the pediatric suicide rate in this country increased $18 \%$ between the years of 2003-2004 [4], signifying the largest single-year increase since 1990. Preliminary findings from national fatal injury data available for 2004-2005 show a continuation of this alarming trend [5], and suggest the possibility of youth suicide as an escalating public health crisis. Therefore, it is increasingly important for health care professionals to identify and intervene with youth at high risk for suicide.

Existing research has highlighted characteristics of youths at high risk for suicide. The overwhelming majority of youth who make suicide attempts demonstrate mood psychopathology, with depression being the most prevalent disorder. Adolescent depression, marked by hopelessness, severe and pervasive suicidal ideation, is a significant contributor to suicidal behavior [6]. The risk for suicide among adolescents with bipolar disorder is even higher [7-9]. In addition, adolescent males have higher rates of suicide than do adolescent females, who typically report higher rates of suicidal ideation and have higher rates of

(C) 2011 Society for Adolescent Medicine. Published by Elsevier Inc. All rights reserved.

Publisher's Disclaimer: This is a PDF file of an unedited manuscript that has been accepted for publication. As a service to our customers we are providing this early version of the manuscript. The manuscript will undergo copyediting, typesetting, and review of the resulting proof before it is published in its final citable form. Please note that during the production process errors may be discovered which could affect the content, and all legal disclaimers that apply to the journal pertain. 
suicidal behavior [3]. Consistent with adult studies [9-10], a growing body of research suggests that sexual minority youth (SMY; youth who endorse same-sex attraction, samesex behavior, or a gay/lesbian identity) are also at increased risk for mood disorders and suicidality [11-14]. The primary aim of this paper was to summarize, describe, and compare rates of suicidality and depression between SMY and heterosexual youth.

Minority stress theory suggests that disparities between sexual minority and heterosexual youth can be attributed in part to stigma, discrimination, and victimization experiences that are a result of a homophobic and violent culture [15]. Among the factors that researchers have found to be associated with psychosocial risks in SMY are others' negative responses to gender atypical behavior, high-risk sexual behavior, conflicts related to disclosure of sexual orientation to family and its consequences, and mistreatment in community settings, especially schools [16]. One or more of these stressors can promote feelings of helplessness and hopelessness that may develop into depression and suicidality.

Despite the robust empirical and theoretical evidence for higher rates of depression and suicidality among SMY, the size of these disparities varies across studies, warranting a systematic investigation into the potential sources of heterogeneity. For example, evidence suggests that the disparities may vary across: gender [13, 17, 18], bisexuality status [19, 20], and different measures of sexual orientation (e.g., same-sex sexual behavior [21] versus identity labels such as "gay" and "lesbian" [22]). Previous research has shown that these and other sample and study characteristics moderate the association between sexual orientation and outcomes such as substance use and abuse [23]. Thus, another goal of this paper is to examine whether or not these variables moderate suicidality and depression outcomes, in order to corroborate and expand on the existing literature.

Suicidality and depression effect sizes may vary as a function of how the constructs are measured. Effect sizes may vary based on whether or not researchers measure depression using well-developed depression scales or single-item depression measures. Furthermore, SMY disparities may vary depending on the severity of the suicidality or how suicidality is operationalized. For example, some studies have examined disparities in suicidal ideation [20], whereas others have examined a wider range of suicidal behaviors including suicide attempts requiring medical attention [21, 22]. Finally, questions remain regarding whether or not disparities persist after controlling for potential confounding variables. For example, as teenagers get older they are more likely to endorse a same-sex orientation and more likely to endorse depression symptoms, suggesting that age may act as a confounder that accounts for part or all of the disparity.

In sum, the primary goal of this study was to summarize and describe suicidality and depression disparities between SMY and heterosexual youth. The second goal was to determine whether or not methodological characteristics of the original studies and sample characteristics explained variability in the disparities observed across studies including gender, bisexuality status, and how sexual orientation, depression, and suicidality were operationalized. The third goal was to review the methodological qualities of this literature in order to determine how many original studies examined longitudinal patterns of suicidality and depression, as well as mediators, moderators, and potential confounders of the association between sexual orientation and the outcomes.

\section{Method}

Meta analysis reporting guidelines [24] developed and recommended by the Centers for Disease Control and Prevention were followed closely for this study. 


\section{Selection of Studies}

There were two criteria for the inclusion of studies in the meta-analyses: 1) reported rates of depression and/or suicidality among sexual minority and heterosexual youth; and 2) a sample mean age of 18 or less, and an upper bound of the age range not exceeding 21 years. These age criteria were used to insure that the majority of participants in the original studies were adolescents. Studies were identified for the analysis in four steps. First, a systematic search of PsychInfo and MedLine was conducted to identify all eligible studies (published in 2009 or earlier) using various combinations of key terms including: "suicide," "depression," "gay," "lesbian," "LGB," "adolescent," and others. A total of 378 abstracts were identified and reviewed to determine their eligibility (the majority of ineligible studies were excluded because they either focused on youth ages 18-25 years old, did not include a heterosexual comparison group, or they were review papers). Second, papers were retrieved and reviewed to confirm their eligibility $(n=30)$. Third, all eligible studies were reviewed to identify additional studies. Finally, letters were mailed to the corresponding authors of eligible studies asking for their help in identifying unpublished studies that met our inclusion criteria. One additional study was identified by this method [25]. A total of 20 suicide [11-14, 17-22, 25-34] and 12 depression [14, 17, 20, 22, 26, 27, 31, 35-38] studies were identified, resulting in 24 total with 7 studies examining both outcomes.

\section{Coding of Studies}

Pertinent qualitative and quantitative data were extracted from the included studies which fell into four categories: 1) definition of sexual orientation; 2) depression and suicide measures; 3) moderating variables (e.g., bisexuality status; gender); and 4) the effect size data. Two co-authors coded all data. Coders achieved $100 \%$ agreement on all qualitative data. There were 727 individual pieces of data associated with effect size estimates (e.g., sub-sample sizes, p-values, t-test values, etc.). The intra-class correlation between raters of the effect size data was high (.96), and inter-rater agreement was 85\%. Discrepancies between raters were resolved by consensus among the two raters and the first author.

Operationalization of sexual orientation-Four coding categories were used, including measures of: 1) self-identification as gay, lesbian, or bisexual, 2) same-sex romantic or sexual attraction, 3) same-sex romantic or sexual behavior, and 4) two or more of categories 1-3.

Bisexuality status-Participants' bisexuality status was indicated in three ways: 1) selfreported labeling as bisexual, 2) self-reported romantic or sexual attraction to both sexes, or 3) reporting a history of sexual behavior with both sexes.

Suicidality-Suicidality included the participants' reports of: (1) suicidal ideation (thoughts about suicide); (2) suicidal plans or intent; (3) suicide attempts; and (4) suicide attempts that caused injury and/or required medical attention. Furthermore, we distinguished between studies that operationalized the variables as recent suicidality (occurred within the previous year) versus lifetime suicidality.

Depression outcome variables-We distinguished between studies that used singleitem indicators of depression (e.g., "During the past week, how often did you feel depressed?" [20]) and those that used multiple item measures, such as the CESD [39] or the Beck Depression Inventory [40]. One study reported rates of Major Depressive Disorder which was assessed using a comprehensive diagnostic interview [31]. 


\section{Data Analysis Plan}

The data analysis proceeded in several steps. First, overall effects for suicide and depression outcomes were estimated by combining weighted effects across all studies assuming a random effects model (based on rationale provided by Borenstein and colleagues [41]). Second, methodological characteristics were tested as moderators of the overall effect by estimating a "Q" statistic that tests for heterogeneity across moderator subgroups. Mixedeffects models were used for the subgroup analyses such that a random-effects model was assumed when computing summary effects within subgroups, and the overall summary effect (across subgroups) was recalculated by combining the subgroup effects assuming that the subgroup categories were fixed [41]. Third, outcome variables were categorized based on how suicide was operationalized (e.g., ideation versus attempt) and the time frame of use (recent versus lifetime), and the association between sexual orientation and these different variables were estimated. Fourth, subanalyses were conducted in order to examine how the inclusion of covariates impacted the overall estimates. Fifth, diagnostics were performed in order to identify potential outliers, publication biases, and other threats to the statistical conclusion validity of the results. We also compared the average effect size estimates for groups of studies that did and did not employ a public use data set in their analyses in order to examine the effects of large sample sizes on the overall results.

Data management and analyses were conducted using software developed by the National Institutes of Health (Comprehensive Meta Analysis, Version 2) [42]. In most of the studies the suicide outcome variables were categorical, thus the suicide meta-analysis results are reported using an odds-ratio effect size metric. In most of the original studies the depression outcome variables were continuous; thus the depression meta-analysis results are reported using a standardized mean difference effect size metric (Cohen's d; [43]).

\section{Results \\ Suicidality}

The literature search yielded a total of 20 suicidality studies with 122 corresponding effect size estimates. Studies reported multiple effect size estimates due to having multiple outcome variables, multiple demographic subgroups, or both. One effect size was over 5 standard deviations larger than the overall weighted effect size [30] therefore was excluded from the analyses. Furthermore, 16 out of the original 122 effects were redundant with other effects within the individual studies (e.g., some studies reported effects for boys and girls separately and combined. We retained the effects that facilitated our ability to examine subgroup differences). Removing the outlier and redundant effect sizes resulted in a total of 19 studies and 105 effect size estimates used in the analyses.

Weighted effect size estimates and methodological characteristics for each suicide study included in the analysis are summarized in Figure 1 and Table 1. Four of these studies used the same two data sets for their analyses [11, 12, 18, 34]: The 1995 Massachusetts and Vermont Youth Risk Behavior Surveys (YRBS). Three other studies used the National Longitudinal Study of Adolescent Health (Add Health) data [14, 20, 27]. These data were combined and analyzed using methods to account for their inter-dependency; hence their combined effect sizes (one for YRBS and one for Add Health) are presented in Figure 1.

Results showed that the estimate for the overall weighted effect size for the relationship between sexual orientation and suicidality was $\mathrm{OR}=2.92(\mathrm{CI}=2.11-4.03)$ and significantly different from zero ( $\mathrm{z}=6.48, \mathrm{p}<.0001)$. Study-level effect sizes ranged from 1.39 [17] to 8.62 [11]. Individual effect sizes ranged from 0.90 [19] to 15.19 [11]. Over $40 \%$ of the individual odds ratios (43/105) were smaller than 2.0, and over $25 \%$ (28/105) were larger than 4.0. Only one of the 105 individual odds ratios was smaller than 1.00 . When the overall effect 
was re-calculated with each study removed, the re-estimated effect sizes ranged from 2.67 to 3.11. Regardless of which study was removed, all of the overall tests remained significant ( $\underline{\text { 's }}<.0001)$. Begg and Mazumdar's rank correlation test $(\mathrm{p}=.70)$, and Egger's linear regression test $(\mathrm{p}=.63)$ suggested that there was not a significant relationship between the standard errors and the effect sizes. Rosenthal's Fail-safe $\mathrm{N}$ test suggested that 2,325 missing studies with null effects would be needed in order to increase the overall p-value to above . 05 .

Estimation of effects with and without covariates-There was variability across studies regarding whether or not they ran multivariate analyses and reported estimates for the relationship between sexual orientation and suicidality adjusted statistically for various demographic and risk and protective factors (e.g., age, gender, race, depression, substance use, etc.). Thirteen studies [11-13, 17, 20-22, 25-27, 29, 31, 33] reported unadjusted effect sizes only, 1 study [18] reported adjusted effects size only, and 5 studies [14, 19, 28, 32, 34] reported both adjusted and unadjusted effect sizes. In order to determine whether or not adjusting for covariates had a significant impact on the overall weighted effect size estimate we conducted a series of subanalyses. The overall weighted effect using only the unadjusted results from these 5 studies combined with the remaining 13 studies with unadjusted effects was $\mathrm{OR}=3.15$ ( $\mathrm{CI}=2.25-4.43)$. The overall weighted effect using only the adjusted effects from the studies that provide them (6 in all) combined with the remaining 13 studies was $\mathrm{OR}=2.82(\mathrm{CI}=2.02-3.93)$. Because many of the covariates that were employed in the original studies could be conceptualized as mediators (e.g., substance use, depression symptoms) rather than confounding variables, the overall estimated effect may be biased low when using only the adjusted coefficients. Therefore, results herein are reported from models that retained both unadjusted and adjusted effects $(\mathrm{OR}=2.92, \mathrm{CI}=2.11-4.03)$.

\section{Moderation of the association between sexual orientation and suicidality-}

Results suggested that bisexuality status was a significant moderator $(\mathrm{Q}=9.7, \mathrm{df}=1, \mathrm{p}<.01)$. Three studies reported effect size estimates for bisexual SMY, four studies reported effect size estimates for non-bisexual SMY, and 15 studies reported results combining bisexual and non-bisexual SMY. Two studies in the "combined" group used the 1995 Massachusetts YRBS [11, 19] and two used the Add Health data [14, 27]. Furthermore, one study using Add Health data [20] and one study using the 1995 Vermont and Massachusetts YRBS data [34] examined the bisexual and non-bisexual SMY groups separately. In order to avoid dependency in the data across moderator subgroups we removed the Add Health and 1995 YRBS studies that combined bisexual and non-bisexual SMY in their original analyses [11, $14,19,27]$. Results showed that the association between sexual orientation and suicidality was strongest among bisexual SMY $(\mathrm{OR}=4.92, \mathrm{p}<.0001, \mathrm{CI}=2.82-8.59)$ and weakest among non-bisexual SMY (OR=1.87, $\mathrm{p}<.0001, \mathrm{CI}=1.35-2.58)$. The $95 \%$ confidence interval for the combined group overlapped with the confidence intervals from the other two $(\mathrm{OR}=3.08, \mathrm{p}<$. 0001, $\mathrm{CI}=2.08-4.57$ ). The overall effect combining the groups and adjusting for the group differences using a mixed effects analysis was $\mathrm{OR}=2.64(\mathrm{p}<.0001, \mathrm{CI}=2.12-3.27)$. None of the following variables moderated the association between sexual orientation and suicidality: (a) definition of sexual orientation, (b) gender, and (c) use of a public data set.

Results stratified by how suicide was operationalized-The definition of suicide (e.g., ideation versus attempt) was not tested as a moderator due to the non-independence of the data; however, the average effect for each definition of suicide was estimated in order to examine their potential influence on effect size variability. Results suggested that disparities in rates of suicidality increased as the severity of the suicidal behavior increased (ideation, $\mathrm{OR}=1.96, \mathrm{n}=9$; intent/plans, $\mathrm{OR}=2.20, \mathrm{n}=4$; attempts, $\mathrm{OR}=3.18, \mathrm{n}=14$; and attempts resulting in injury or medical attention, $\mathrm{OR}=4.17, \mathrm{n}=5$ ). Finally, a trend suggested that the 
association was stronger when recent suicidality was measured $(\mathrm{OR}=3.34)$ as compared with lifetime measures $(\mathrm{OR}=1.92)$.

Summary of the absolute rates of suicidality-Thirteen out of the 19 studies (68\%) reported data that allowed for an estimation of the absolute rates of suicidality in each group, thus these estimates are not representative of the population of studies and participants. However, in order to provide a context for interpreting the meta-analysis results we present the average absolute rates across suicide constructs (e.g., ideation, plans) and across subgroups for each study in Table 1 . The average of these rates showed that $28 \%$ of SMY and $12 \%$ of heterosexual youth reported a history of suicidality. When we averaged the absolute rates across the six studies that reported rates for each gender, we found that: (a) $28 \%$ of sexual minority boys and $17 \%$ of heterosexual boys reported a history of suicidality; and (b) $37 \%$ of sexual minority girls and $23 \%$ of heterosexual girls reported a history of suicidality.

\section{Depression: Overall effect size estimates}

The literature search identified 12 depression studies with a total of 51 corresponding effect size estimates. Fourteen of these effects were redundant with other effects within the individual studies. The effect size for one study [30] was considered an outlier and removed from the analyses because it was over 10 standard deviations larger than the overall weighted average effect size. Thus, a total of 11 studies and 36 effect size estimates were used for the analyses. Weighted effect size estimates and methodological characteristics for each depression outcome study included in the analysis are summarized in Figure 2 and Table 2. Four of the depression studies used the Add Health data set for their analyses [14, $20,27,36]$. These data were combined and analyzed using methods to account for their inter-dependency; hence their combined effect size is presented in Figure 2.

Results showed that the estimate for the overall weighted effect size for the relationship between sexual orientation and depression was $\mathrm{d}=.33$ (95\% CI=.22-.43, $\mathrm{z}=6.02, \mathrm{p}<.0001)$. Mean effect sizes for each study ranged from .17 [37] to .67 [26], and the mean effect size for the Add Health studies combined was .25. Individual effect sizes ranged from -.28 [36] to 1.0 [36]. Approximately $17 \%(6 / 36)$ of the individual effect sizes were smaller than .20 and $17 \%(6 / 36)$ were larger than .50 .

When the overall effect was re-calculated with each study removed, the re-estimated effect sizes ranged from .28-.36. Regardless of which study was removed the overall tests of significance remained significant ( $\mathrm{p}$ 's<.0001). Begg and Mazumdar's rank correlation test $(\mathrm{p}=.22)$, and Egger's linear regression test ( $\mathrm{p}=.18)$ suggested that there was not a significant relationship between the standard errors and the effect sizes. Rosenthal's Fail-safe $\mathrm{N}$ test suggested that 228 missing studies with null effects would be needed in order to increase the overall p-value to above .05 .

Estimation of effects with and without covariates-Two studies examined the association between sexual orientation and depression outcomes controlling for covariates $[27,36]$. One study reported a significant three-way interaction between sexual orientation and two covariates [36] thus its conditional main effects of sexual orientation were not considered. The other study only reported adjusted effect size estimates [36]. The association between sexual orientation and depression did not change when this study was removed from the analysis.

Moderation of the association between sexual orientation and depressionThe nature of the depression measure was a significant moderator $(\mathrm{Q}=3.97, \mathrm{df}=1, \mathrm{p}<.05)$ of 
the association between sexual orientation and depression. One Add Health study employed a single-item measure of depression [20] and three Add Health studies employed the CESD depression scale $[14,27,36]$. We removed the Add Health study that employed a singleitem depression measure in order to avoid dependency in the data across moderator subgroups. Results showed that on average the strongest associations between sexual orientation and depression were found in studies that employed single-item measure of depression $(\mathrm{d}=.50, \mathrm{p}<.0001, \mathrm{CI}=.25-.76)$. The average effect for studies that employed a depression scale such as the CESD or the BDI was $d=.24$ ( $p<.0001, C I=.19-.29)$. The overall effect combining the groups and adjusting for group differences using a mixed effects analysis was $\mathrm{d}=.25(\mathrm{p}<.0001, \mathrm{CI}=.20-.30)$. None of the following variables moderated the association between sexual orientation and depression: definition of sexual orientation, bisexuality status, gender of the participant, and use of public data set.

\section{Discussion}

This study provides strong evidence that SMY experience significantly higher levels of suicidality and depression symptoms than do heterosexual youth. The robust pattern of results, particularly with regards to suicidality, highlights the severity and pervasiveness of disparities between SMY and heterosexual youth. For example, on average 28\% of SMY reported a history of suicidality as compared to $12 \%$ of heterosexual youth. Second, 104 of the 105 individual odds ratios for the association between sexual orientation and suicidality were greater than 1.00 , and over $25 \%$ of them were larger than 4.00 . Third, the disparities increased in size as the severity of the suicidality increased. Finally, studies showed that even after controlling for important explanatory variables, SMY were still almost twice as likely to report a history of suicidality as heterosexual youth.

These results are consistent with the growing number of studies showing that SMY are at risk for developing psychosocial problems. For example, meta-analysis results have shown that sexual minority adults [9] and youth [23] report higher rates of substance use and abuse than do heterosexuals. Recent results show that compared with heterosexual youth, SMY report higher rates of sex under the influence of alcohol or drugs [44]. Furthermore, evidence suggests that SMY, and sexual minority boys in particular, were more likely to have a history of eating disorders than were heterosexual youth [45], a diagnosis associated with significant mood comorbidity and mortality. Finally, recent meta-analysis results showed that compared with heterosexual youth, SMY report higher rates of violence and victimization [46].

Bisexuality was a significant moderator in this study. SMY who were bisexual reported being almost 500\% more likely to report suicidality than were heterosexual youth. SMY who were not bisexual reported being $170 \%$ more likely to endorse suicidality than were heterosexual youth. These results are consistent with previous reviews showing bisexual youth are at greater risk for substance use [23], and suggest that bisexuality status among SMY is particularly stressful phase of sexual identity development. The remainder of the moderation results showed that gender, recruitment source, and operationalization of sexual orientation did not significantly impact the association between sexual orientation and adolescent depression or suicidality. Given that several of these constructs were associated with substance use disparities [23] or victimization disparities among SMY [46], and that there are notable trends in the literature with regard to rates of suicidality across gender, these null results were unexpected. One potential explanation, especially for the depression analysis, is reduced power [41] due to a relatively small number of studies.

Another striking trend in our results was that as the severity of the suicidal behavior increased, the disparity between SMY and heterosexual youth increased. Reasons for this 
trend remain unclear; however high rates of violence and victimization among SMY [46] might give rise to higher levels of hopelessness, increasing the likelihood of an attempt. High rates of substance use [23] may also increase the likelihood of suicide attempts. This trend suggests that clinicians and caregivers should pay close attention to the early signs of suicidality among SMY and intervene early to prevent more serious suicidal behaviors from developing.

There are several important limitations of the literature. First, none of the studies examined individual trajectories of depression or suicidality into young adulthood. Two recent longitudinal studies found that sexual orientation in adolescence predicted suicidality in young adulthood $[47,48]$. Future studies are needed that can examine individual trajectories of mental health problems over time, to confirm that for high risk adolescents, problems may persist and even grow more severe as they transition into young adulthood. For example, individual trajectory modeling has been used to describe change in substance use among SMY as they transition into young adulthood [49-52].

Second, five studies reported components of the "causal steps" approach to testing mediation [53], by estimating the association between sexual orientation and suicidality before and after controlling for potential confounders or mediators [14, 19, 28, 32, 34]. However, only one study [38] tested a full mediation model using a product of coefficients test [54], providing the strongest evidence to date that victimization significantly mediated the association between sexual orientation and depression. Future studies that can provide similar evidence for mediators will help inform theory and identify targets for prevention and intervention programs.

Third, only three studies examined moderators of these disparities between SMY and heterosexual youth $[11,27,36]$. Bontempo and D'Augelli [11] found that rates of suicidal ideation were higher among SMY who reported a history of victimization. These results suggest that victimization experiences play a key role in the mental health problems of SMY. Future studies that identify demographic and substantive moderators of this disparity will help researchers tailor their prevention and intervention programs to these high risk subgroups.

\section{Clinical Implications for Adolescent Mental Health Services}

The biggest challenge facing mental health service professionals is identifying adolescents most at-risk for suicidal events. Results from this meta-analysis provide strong evidence that SMY are at a substantially heightened risk for suicide and depression. Mental health service professionals who assess and treat adolescents may wish to pay particular attention to patients who identify as lesbian or gay or who endorse same-sex attraction, as these youths may face unique or severe negative circumstances that exacerbate depression. SMY presenting with depression should be screened carefully for past and current suicidal thoughts and attempts, and risk factors for suicidal behavior such as substance use and abuse. A detailed plan for maintaining safety should be established even in the absence of current suicidality, in the effort to decrease impulsive self-harm. Finally, clinicians should be prepared to promote healthier outcomes among SMY by advocating and promoting no tolerance policies in schools to help prevent bullying and peer victimization, protective factors such as positive relationships with family and connection with friends $[55,56]$, and resources in schools for youth including gay-straight alliance organizations and on-site mental health providers.

Depression in SMY should be treated aggressively with empirically supported interventions in order to decrease the likelihood of suicidal behaviors. Current treatment guidelines include the combination of selective serotonin reuptake inhibitors (SSRI) and empirically 
supported psychotherapies, such as cognitive behavior therapy (CBT) or interpersonal psychotherapy for depressed adolescents (IPT-A); However, extant treatment studies for youth depression have not provided information regarding the efficacy of psychotherapies or SSRIs in SMY. Future treatment studies for youth depression should also collect information on sexual orientation in order to provide more specific outcome data in SMY, to tailor empirically supported interventions to the unique needs of SMY and their families.

In sum, our results showed that SMY are at increased risk for suicidality and depression, and that these disparities are strong and pervasive, remaining significant in multiple subpopulations after taking into account other risk and protective factors. The identification of significant moderator variables in our results can help the design of future studies that can identify explanatory mechanisms of significant moderators such as bisexuality status. Future studies should also focus on articulating and testing longitudinal and mediated pathways of risk among SMY, in order to identify key mechanisms that can be targeted by prevention and intervention programs. Finally, clinicians are encouraged to promote a safe and confidential environment for SMY to discuss their orientation with their health care providers, and assess depression and suicidality adequately in order to provide appropriate care for youth in need [57].

\section{References}

1. Bridge JA, Goldstein TR, Brent DA. Adolescent suicide and suicidal behavior. J Child Psychol and Psyc. 2006; 47(3):372-394.

2. Eaton D, Kahn L, Kinchen SA, et al. Youth risk behavior surveillance - United States, 2005. Centers for Disease Control and Prevention, National Center for Health Statistics. 2006; 55:1-108.

3. Lewinsohn PM, Rhode P, Seeley JR. Adolescent suicidal ideation and attempts: Prevalence, risk factors, and clinical implications. Clinical Psychology: Science and Practice. 1996; 3:25-46.

4. [July 12, 2010] Mortality Query, CDC Wonder, Data, and Statistics. 2006. Available at: http:// wonder.cdc.gov/mortSQL.html

5. Bridge JA, Greenhouse JB, Weldon AH, Campo JV, Kelleher KJ. Suicide trends among youths aged 10 to 19 years in the United States, 1996-2005. JAMA. 2008; 300(9):1025-6. [PubMed: 18768413]

6. Brent DA, Birmaher B. Adolescent depression. N Engl J Med. 2002; 347(9):667-671. [PubMed: 12200555]

7. Brent DA. Depression and suicide in children and adolescents. Pediatrics in Review. 1993; 14:380388. [PubMed: 8255818]

8. Goldstein, TR.; Brent, DA. Youth Suicide.. In: Dulcan, MK., editor. Dulcan's Textbook of Child and Adolescent Psychiatry. American Psychiatric Publishing, Inc; Washington, DC: 2009. p. 531-542.

9. King M, Semlyen J, Tai SS, Killaspy, et al. A systematic review of mental disorder, suicide, and deliberate self harm in lesbian, gay and bisexual people. BMC Psychiatry. Aug 18. 2008; 8:70. [PubMed: 18706118]

10. Meyer IH. Prejudice, social stress, and mental health in lesbian, gay, and bisexual populations: conceptual issues and research evidence. Psychol Bull. 2003; 129:674-97. [PubMed: 12956539]

11. Bontempo DE, D'Augelli AR. Effects of at-school victimization and sexual orientation on lesbian, gay, or bisexual youths' health risk behavior. J Adolescent Health. 2002; 30:364-474.

12. Garofalo R, Wolf RC, Kessel S, et al. The association between health risk behaviors and sexual orientation among a school-based sample of adolescents. Pediatrics. 1998; 101(5):895-902. [PubMed: 9565422]

13. Remafedi G, French S, Story M, et al. The relationship between suicide risk and sexual orientation: Results of a population-based study. Am J Public Health. 1998; 88(1):57-60. [PubMed: 9584034]

14. Russell ST, Joyner K. Adolescent sexual orientation and suicide risk: Evidence from a national study. Am J Public Health. 2001; 91(8):1276-1281. [PubMed: 11499118] 
15. Meyer IH. Prejudice, social stress, and mental health in lesbian, gay, and bisexual populations: conceptual issues and research evidence. Psych Bull. 2003; 129:674-697.

16. Rivers, I.; D'Augelli, AR. The victimization of lesbian, gay, and bisexual youths: Implications for intervention.. In: D'Augelli, AR.; Patterson, CJ., editors. Lesbian, gay, and bisexual identities and youths: Psychological perspectives. Oxford University Press; New York: 2001. p. 199-223.

17. Caldwell LL, Kivel BD, Smith EA, Hayes D. The leisure context of adolescents who are lesbian, gay male, bisexual and questioning their sexual identities: An exploratory study. J Leisure Res. 1998; 30(3):341-355.

18. Garofalo R, Wolf C, Wissow LS, et al. Sexual orientation and risk of suicide attempts among a representative sample of youth. Arch Pediatr Adolesc Med. 1999; 153:487-493. [PubMed: 10323629]

19. Olshen E, McVeigh KH, Winsch-Hitzig RA, et al. Dating violence, sexual assault, and suicide attempts among urban teenagers. Arch Pediatr Adolesc Med. 2007; 161:539-545. [PubMed: 17548757]

20. Udry J, Chantala K. Risk assessment of adolescents with same-sex relationships. J Adol Health. 2009; 31:84-92.

21. Faulkner AH, Cranston K. Correlates of same-sex sexual behavior in a random sample of Massachusetts high school students. Am J Public Health. 1998; 88(2):262-266. [PubMed: 9491018]

22. Safren SA, Heimberg RG. Depression, hopelessness, suicidality, and related factors in sexual minority and heterosexual adolescents. J Consult Clin Psych. 1999; 67(6):859-866.

23. Marshal MP, Friedman MS, Stall R, et al. Sexual orientation and adolescent substance use: A meta-analysis and methodological review. Addiction. 2008:103,546-556.

24. Stroup D F, Berlin J A, Morton SC, et al. Meta-analysis of observational studies in epidemiology: a proposal for reporting. JAMA. 2000; 283:2008-12. [PubMed: 10789670]

25. Smith, A.; Steward, D.; Peled, M.; Poon, C.; Saewyc, E.; the McCreary Centre Society. A Picture of Health: Highlights from the 2008 BC Adolescent Health Survey. McCreary Centre Society; Vancouver, BC: 2009.

26. Barney DD. Health risk-factors for gay American Indian and Alaska Native adolescent males. J Homosexuality. 2003; 46(1/2):137-157.

27. Consolacion TB, Russell ST, Sue S. Sex, race/ethnicity, and romantic attractions: Multiple minority status adolescents and mental health. Cult Divers Ethn Min. 2004; 10(3):200-214.

28. Eisenberg ME, Resnick MD. Suicidality among gay, lesbian and bisexual youth: The role of protective factors. J Adolescent Health. 2006; 39:662-668.

29. Goodenow C, Szalacha L, Westheimer K. School support groups, other school factors, and the safety of sexual minority adolescents. Psychol Schools. 2006; 43(5):573-589.

30. Lampinen TM, McGhee D, Martin I. Increased risk of "club" drug use among gay and bisexual high school students in British Columbia. J Adolescent Health. 2006; 38:458-461.

31. Noell JW, Ochs LM. Relationship of sexual orientation to substance use, suicidal ideation, suicide attempts, and other factors in a population of homes adolescents. J Adolescent Health. 2001; 29:31-36.

32. Pinhey TK, Millman SR. Asian/Pacific Islander adolescent sexual orientation and suicide risk in Guam. Am J Public Health. 2004; 94(7):1204-1206. [PubMed: 15226144]

33. Reis, E.; Saewyc, E. 83,000 youth: Select findings of eight population-based studies as they pertain to anti-gay harassment and the safety and well-being of sexual minority students. Safe Schools Coalition of Washington; Seattle, WA: 1999.

34. Robin L, Brener ND, Donahue SF, Hack T, Hale K, Goodenow C. Associations between health risk behaviors and opposite-, same-, and both-sex sexual partners in representative samples of Vermont and Massachusetts high school students. Arch Pediat Adol Med. 2004; 156:349-355.

35. Cochran BN, Steward AJ, Ginzler JA, Cauce AM. Challenges faced by homeless sexual minorities: Comparison of gay, lesbian, bisexual, and transgender homeless adolescents with their heterosexual counterparts. Am J Public Health. 2002; 92(5):773-777. [PubMed: 11988446] 
36. Galliher RV, Rostosky SS, Hughes HK. School belonging, self-esteem, and depressive symptoms in adolescents: An examination of sex, sexual attraction status, and urbanicity. J Youth Adolescence. 2004; 33(3):235-245.

37. van Griensven F, Kilmarx PH, Jeeyapant S, et al. The prevalence of bisexual and homosexual orientation and related health risks among adolescents in northern Thailand. Arch of Sex Beh. 2004; 33:137-147.

38. Williams T, Connolly J, Pepler D, Craig W. Peer victimization, social support, and psychosocial adjustment of sexual minority adolescents. J Youth Adol. 2005; 34(5):471-482.

39. Radloff LS. The CES-D Scale: A Self-Report Depression Scale for Research in the General Population. Applied Psychological Measurement. 1977; 1(3):385-401.

40. Beck AT, Ward CH Mendelson M, et al. An Inventory for Measuring Depression. Arch Gen Psychiatry. 1961; 4(6):561-571. [PubMed: 13688369]

41. Borenstein, M.; Hedges, L.; Higgins, J.; Rothstein, H. Introduction to Meta-Analysis. Wiley; UK: 2009.

42. Borenstein, M.; Hedges, L.; Higgins, J.; Rothstein, H. Comprehensive Meta-analysis. Biostat; Englewood, NJ: 2005. version 2.

43. Cohen, J. Statistical Power for the Behavioral Sciences. 2nd ed.. Erlbaum; Hillsdale, NJ: 1988.

44. Herrick A, Marshal MP, Smith H, Stall R. Sex While Intoxicated: A Meta-Analysis Comparing Heterosexual and SMY. Journal of Adolescent Health. In press.

45. Austin SB, Ziyadeh NJ, Corliss HL, et al. J Adolesc Health. Sep. 2009; 45(3):238-45. [PubMed: 19699419]

46. Friedman MS, Marshal MP, Guadamuz TE, et al. A Meta-Analysis to Examine Disparities in Childhood Physical and Sexual Abuse Among Sexual and Non-Sexual Minorities. American Journal of Public Health. In press.

47. Russell ST, Toomey RB. Men's sexual orientation and suicide: Evidence for adolescent specific risk. Social Science and Medicine. In press.

48. Wichstrom L, Hegna K. Sexual orientation and suicide attempt: A longitudinal study of the general Norwegian adolescent population. J Abnorm Psychol. 2003; 112(1):114-151.

49. Corliss HL, Rosario M, Wypij D, et al. Sexual orientation disparities in longitudinal alcohol use patterns among adolescents: Findings from the Growing Up Today Study. Arch Pediatr Adolesc Med. 2008; 162:1071-1078. [PubMed: 18981356]

50. Hatzenbuehler ML, Corbin WR, Fromme K. Trajectories and determinants of alcohol use among LGB young adults and their heterosexual peers: results from a prospective study. Dev Psychol. 2008; 44:81-90. [PubMed: 18194007]

51. Marshal MP, Friedman MS, Stall R, Thompson AL. Individual trajectories of substance use in lesbian, gay, and bisexual youth and heterosexual youth. Addiction. 2009; 104:974-981. [PubMed: 19344440]

52. Talley AE, Sher JK, Littlefield AK. Sexual orientation and substance use trajectories in emerging adulthood. Addiction. 2010; 105:1235-1245. [PubMed: 20491728]

53. Baron RM, Kenny DA. The moderator-mediator variable distinction in social psychological research: Conceptual, strategic, and statistical considerations. J Pers Soc Psychol. 1986; 51:11731182. [PubMed: 3806354]

54. MacKinnon DP, Lockwood CM, Hoffman JM, et al. comparison of methods to test the significance of the mediated effect. Psychological Methods. 2002; 7(1):83-104. [PubMed: 11928892]

55. Borowsky IW, Ireland M, Resnick MD. Adolescent suicide attempts: Risks and protectors. Pediatrics. 2001; 107:485-493. [PubMed: 11230587]

56. Resnick MD, Bearman PS, Blum, et al. Protecting adolescents from harm. Findings from the National Longitudinal Study on Adolescent Health. Journal of the American Medical Association. 1997; 278:823-832. [PubMed: 9293990]

57. Coker TR, Austin SB, Schuster MA. The health and health care of lesbian, gay and bisexual adolescents. Annu Rev Public Health. 2010; 31:457-477. [PubMed: 20070195] 


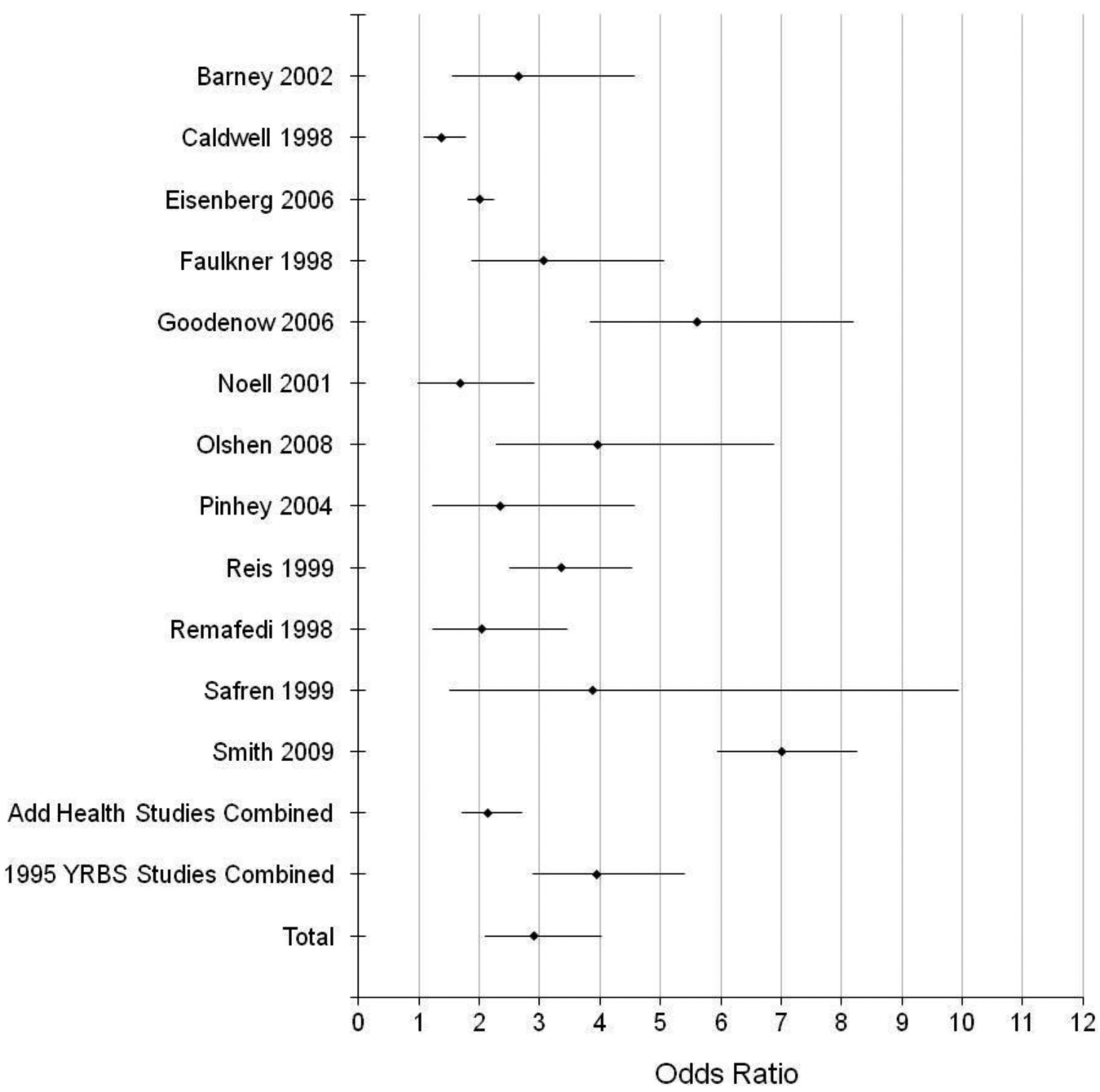

Figure 1.

Odds ratios and $95 \%$ confidence intervals for studies testing the association between sexual orientation and adolescent suicidality. 


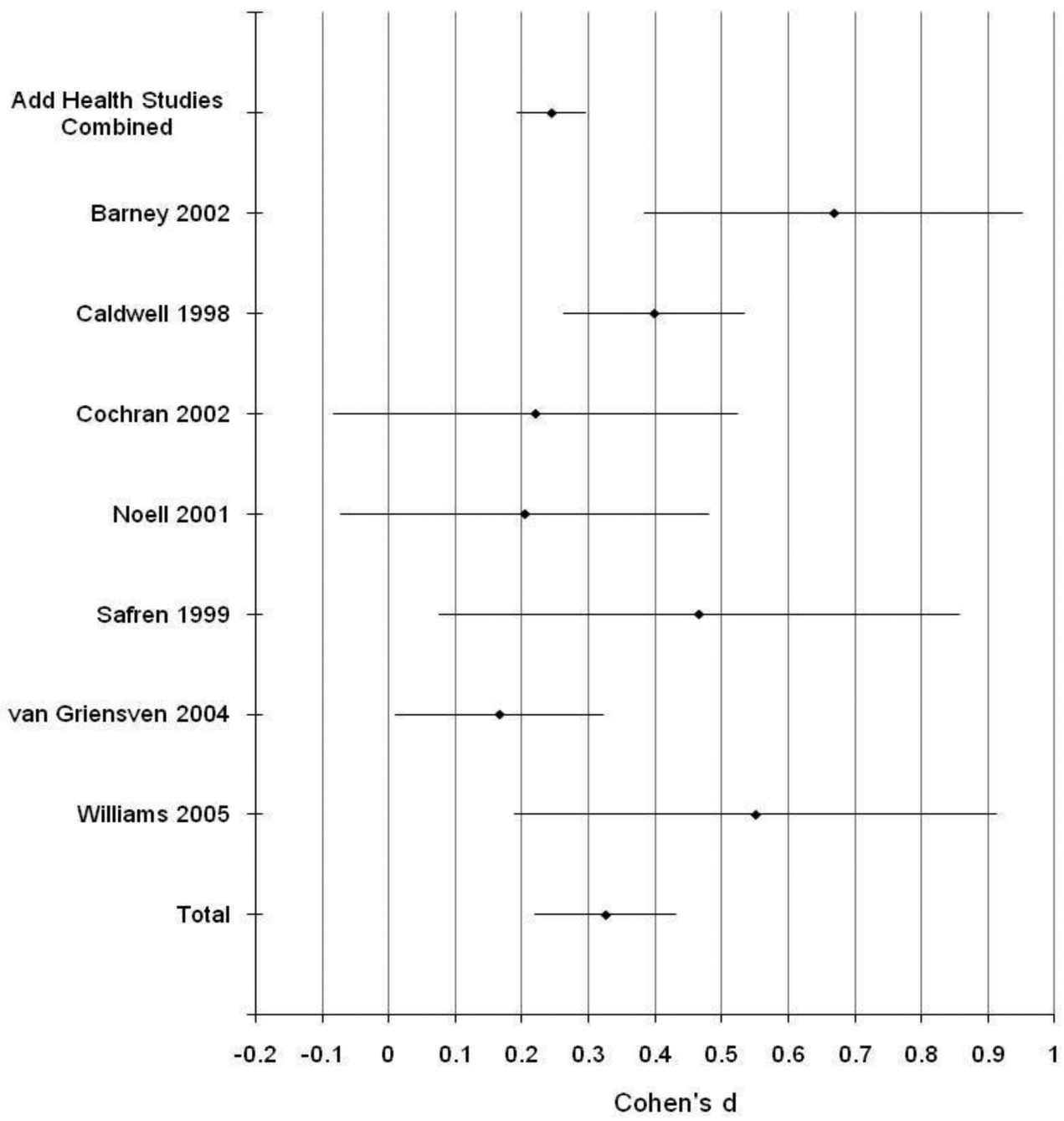

Figure 2.

Cohen's ds and 95\% confidence intervals for studies testing the association between sexual orientation and adolescent depression symptoms. 


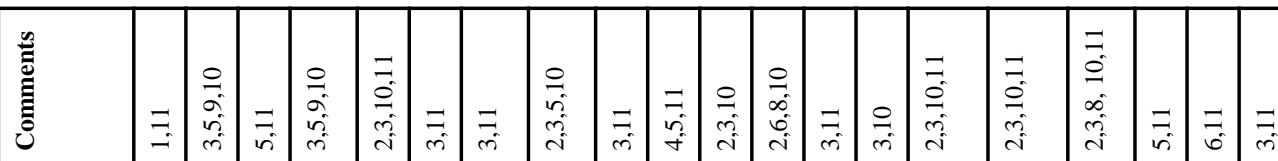

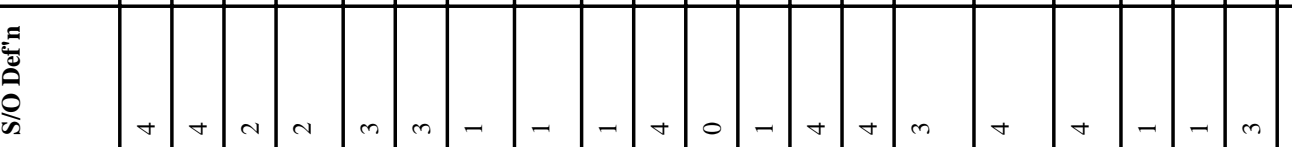

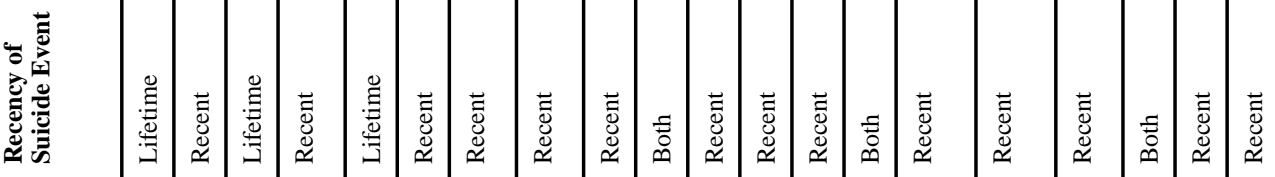

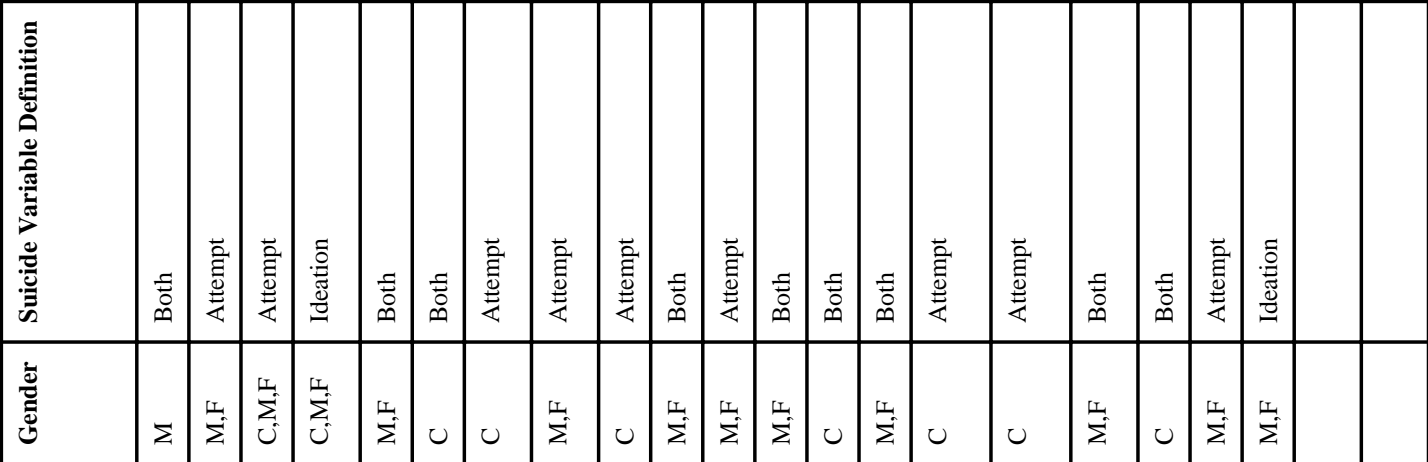

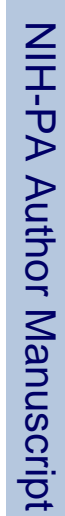

言

(a)

象野

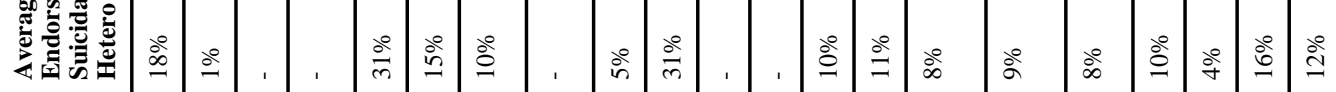

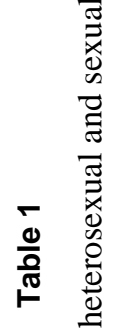

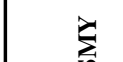

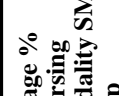

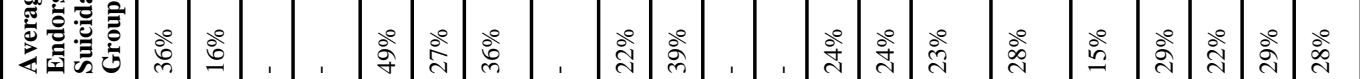

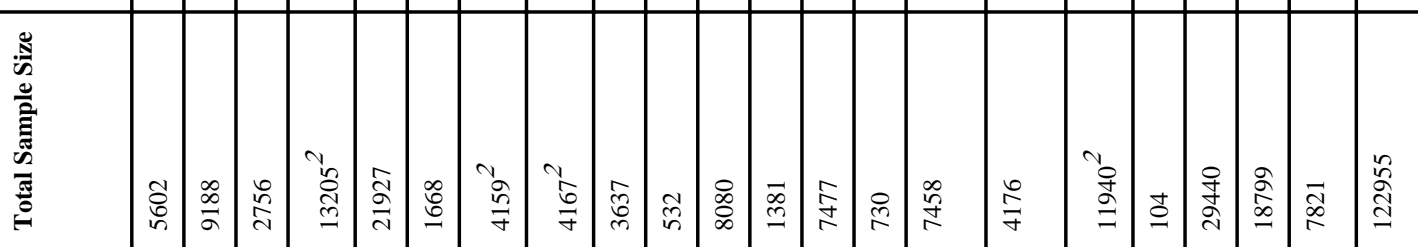

里

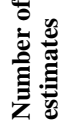

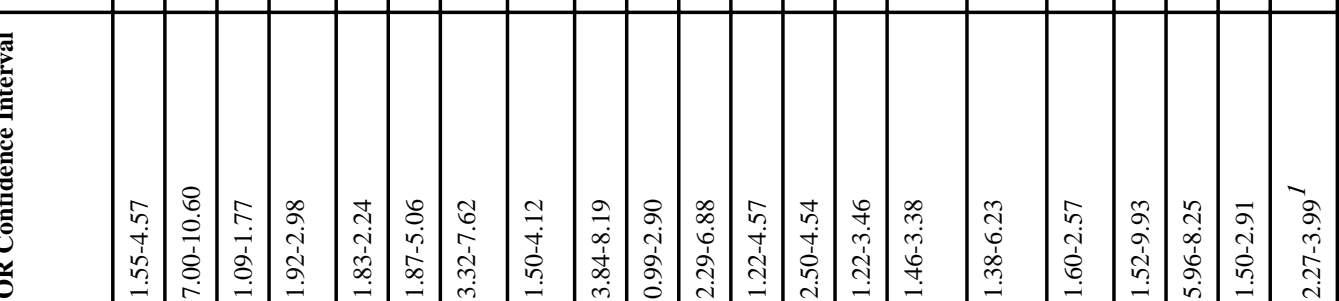

పิ

要

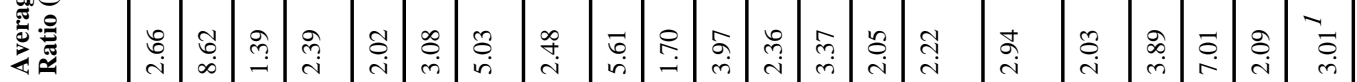

ปี

䓛

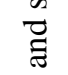

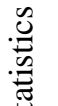

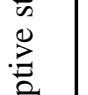

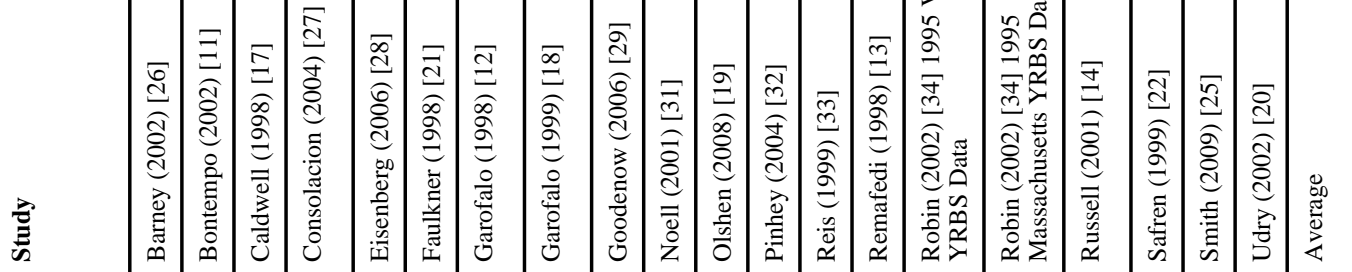

西

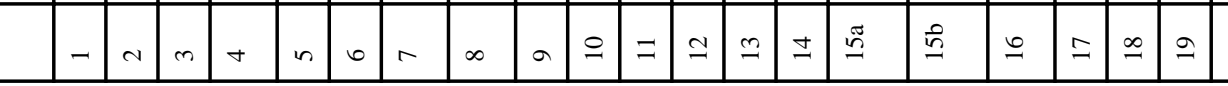



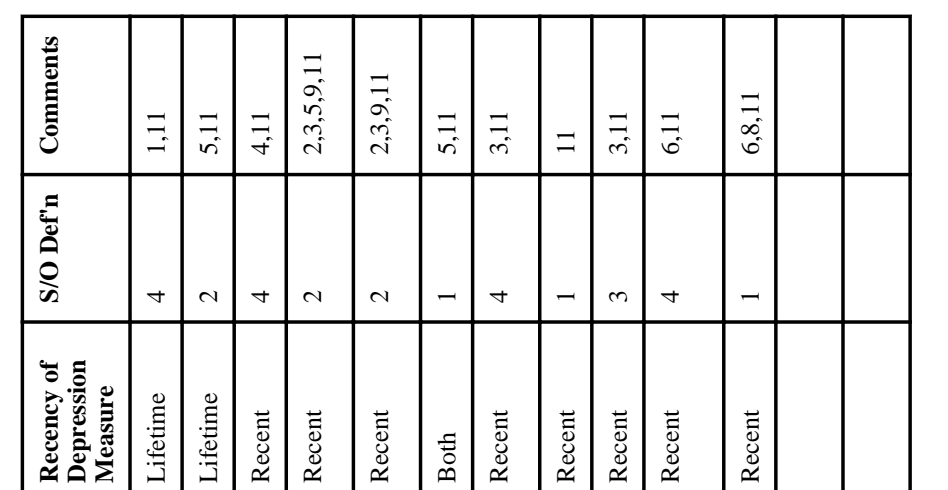

$$
\text { : }
$$

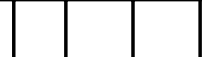

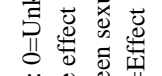

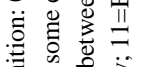

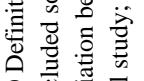

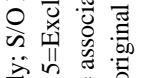

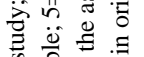

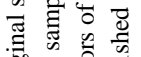

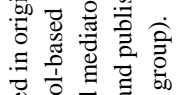

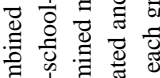

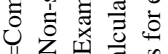

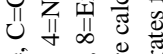

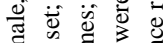

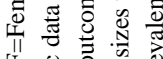

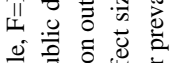

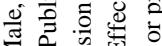

$\sum_{\Sigma}$ il

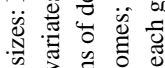

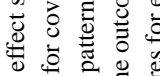

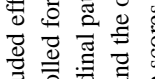

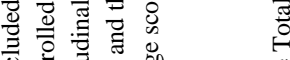

के

II

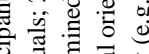

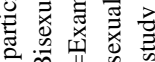

tै

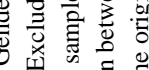

II

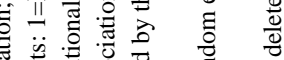

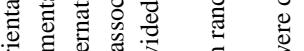

을 हो

要,

की

के

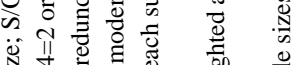

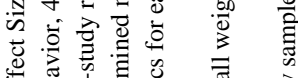

